\title{
CASE REPORT: CONCOMITANT UVEITIS AND LINEAR SCLERODERMA IN AN ADULT PATIENT
}

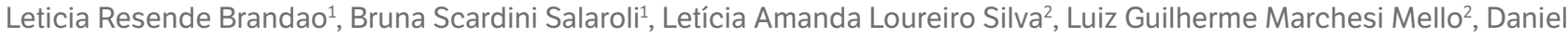
Caiado Fraga Lavagnoli², Luiz Fellipe Favoreto Genelhu' ${ }^{1}$, Elizandra Tomazela Laurenti Polito ${ }^{1, \star}$

1.Hospital Estadual Dorio Silva, Serra (ES), Brazil; 2.Hospital Universitário Cassiano Antonio Moraes, Vitória (ES), Brazil.

*Corresponding author: eliz.polito@gmail.com

\section{BACKGROUND}

We report a case of severe anterior uveitis (AU) and herpes simplex with visual loss and concomitant appearance of linear scleroderma "en coup de sabre" (LSCS).

\section{CASE REPORT}

In 2019, a 38-year-old female presented paresthesia in left hemiface associated with visual blurring of the left eye and mild ocular pain. Concomitantly, she evolved with a frontal lesion, typical of LSCS, atrophic and hyperchromic lesion, with alopecia area on the scalp (Figure 1). She received ophthalmologic emergency care, and disciform keratitis was observed, with AU, and a presumed diagnosis of herpetic endotheliitis. Serology for herpes simplex types 1 and 2, positive IgG. Negative serologies for hepatitis, CMV, HTLV, HIV, syphilis, toxoplasmosis. Empirical treatment with prednisolone $1 \%$ topical and oral acyclovir with good visual recovery. After 2 years, she presented severe recurrence of the ocular condition (Figure 2), being referred to dermatology and rheumatology. Diagnostic of scleroderma was based on clinical examination. The diagnosis of herpetic endotheliitis is predominantly clinical, suggested by recurrence of disciform endotheliitis associated with AU. The pathophysiology is complex, and the participation of $\mathrm{CD} 4+\mathrm{T}$ cells is known. Some previous studies suggest an association between herpes family viruses and the subsequent development of scleroderma. The HSV-IgG antibodies were found at increased levels in patients with scleroderma, in addition to DNA sequence homologies between herpes virus and nuclear antigens, suggesting cross-reaction with some nuclear proteins.
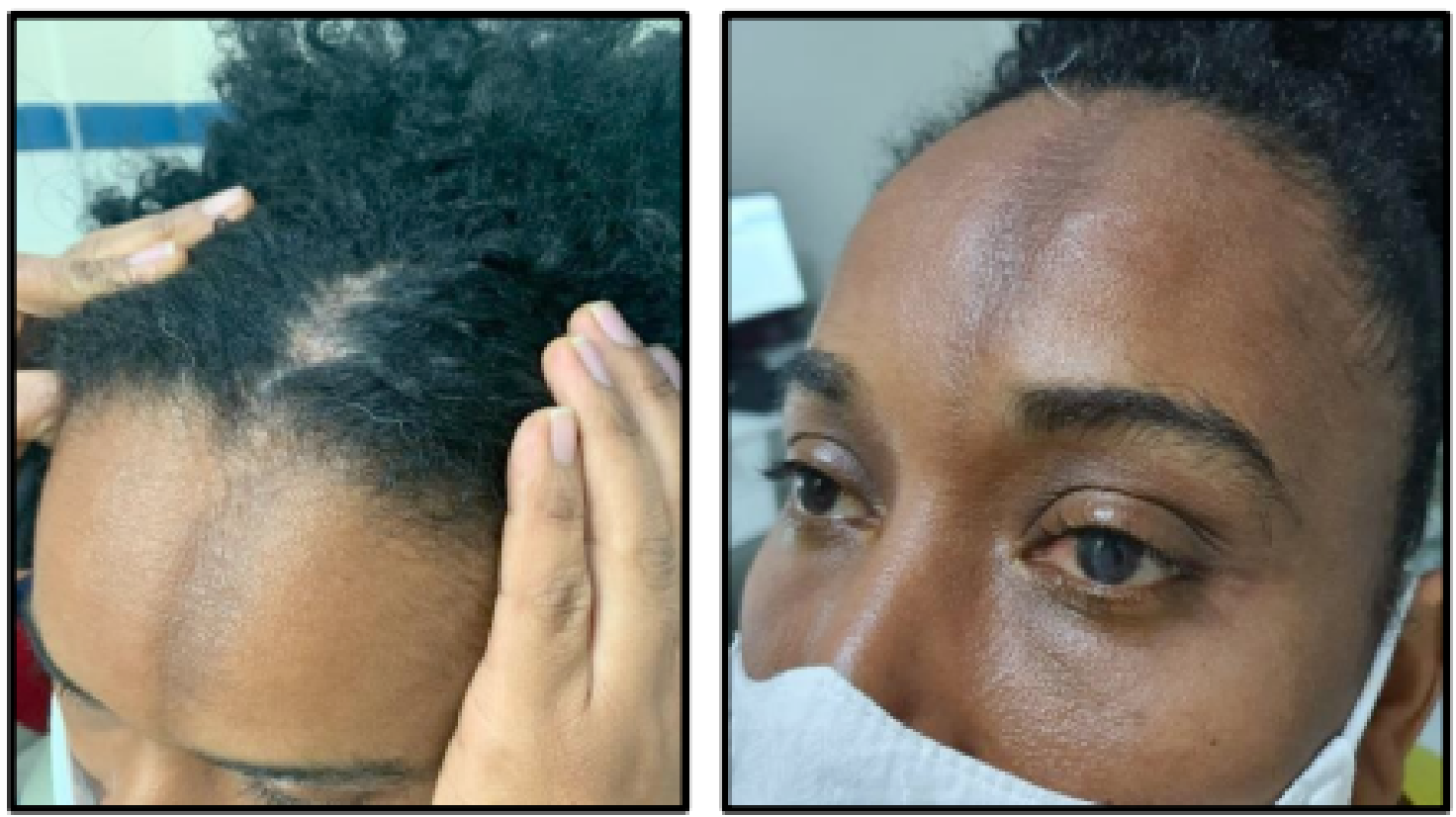

Figure 1. Linear scleroderma "en coup de sabre”. 

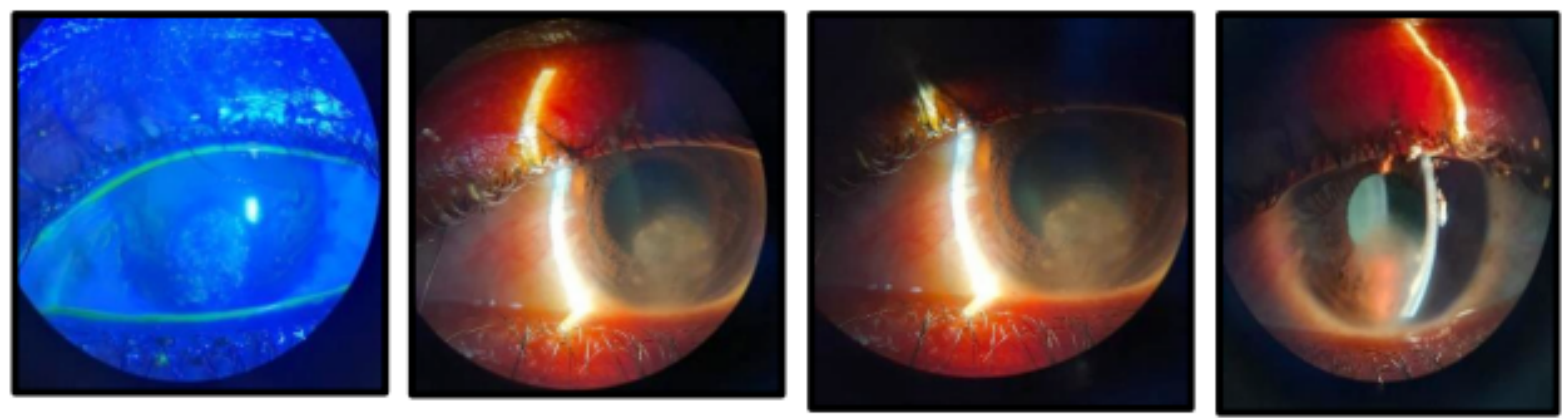

Figure 2. Left eye biomicroscopy in the slit lamp; disciform endotheliitis, with stromal edema of the cornea, folds in Descemet membrane, underlying keratic precipitates. Anterior chamber reaction with cellularity of $3+/ 4+$.

\section{CONCLUSION}

In a review of the literature, we found that LSCS may be associated with extracutaneous manifestations, such as neurological and ophthalmologic symptoms and are more common in the pediatric population. Ophthalmologic involvement occurs almost exclusively in linear forms with cephalic involvement. The most common ocular manifestations are a consequence of the fibrotic process leading to enophthalmia, epiphora, rarefaction or alopecia of the eyelashes and supra lashes and xerophthalmia. The second most frequent group results from inflammatory changes such as AU, episcleritis and keratitis. Anterior uveitis may be asymptomatic, being important ophthalmologic follow-up. For more severe forms of localized scleroderma, immunosuppressors such as methotrexate are recommended. We share a rare case of adult-onset linear scleroderma with anterior herpes simplex-associated uveitis. It is important to recognize the occurrence of LSCS in adulthood and its ophthalmological manifestations of inflammatory character for subsequent initiation of immunosuppressive treatment as a means of prevention of sequel.

\section{KEYWORDS}

Linear scleroderma, Linear scleroderma en coup de sabre, Anterior uveitis, Adult-onset. 